\title{
Gas Sensing with Porphyrin Functionalized Metal Oxide Nanostructures ${ }^{\dagger}$
}

\author{
Gabriele Magna ${ }^{1}$, Mounika Muduganti ${ }^{1}$, Roberto Paolesse 2 and Corrado Di Natale 1,* \\ 1 Department of Electronic Engineering, University of Rome Tor Vergata, 00133 Rome, Italy; \\ magna.gabriele@gmail.com (G.M.); mounika.nano@gmail.com (M.M.) \\ 2 Department of Chemical Science and Technology, University of Rome Tor Vergata, 00133 Rome, Italy; \\ roberto.paolesse@uniroma2.it \\ * Correspondence: dinatale@uniroma2.it \\ + Presented at the 8th GOSPEL Workshop. Gas Sensors Based on Semiconducting Metal Oxides: Basic \\ Understanding \& Application Fields, Ferrara, Italy, 20-21 June 2019.
}

Published: 19 June 2019

In the last years we have been interested to study the interplay of light and gas sensitivity in hybrid materials formed by porphyrins grafted onto $\mathrm{ZnO}$ nanostructures. First results shown that the conductivity of the hybrid material is sensitive to the visible light and to donor-acceptor gases such as amines and alcohols [1]. Surface photovoltage studies of the sensing properties of these materials suggests that the adsorption of volatile compounds may modulate, either positively or negatively, the photovoltaic properties of the dye-semiconductor hybrid [2].

The standard procedure to functionalize $\mathrm{ZnO}$ surface requires the addition of a carboxylic group functionalized porphyrin to a solution where the $\mathrm{ZnO}$ nanostructure is dipped. However, most of the gas sensing properties depend on the surface arrangement of the porphyrin layer. Thus, changes in the molecular film formation procedure may result in different sensors. This feature prompts the development of alternative routes for the hybrid film formation. One-pot methods, where porphyrin is directly added to the $\mathrm{ZnO}$ synthesis solution have been introduced, thus in spite of the same components ( $\mathrm{ZnO}$ and porphyrin) the materials show different morphology and different sensitivity [3].

Further evidence of the importance of the molecular film morphology was obtained with porphyrins coated $\mathrm{ZnO}$ nanoparticles spotted on quartz microbalances (QMBs). In this study, a minimal array formed by the same porphyrin deposited onto three surfaces was considered. The same porphyrin (Zinc tetraphenylporphyrin) was spotted on the bare gold pad of the QMB and on $\mathrm{ZnO}$ nanoparticles. The coating of nanoparticles was obtained functionalizing the nanoparticles and with the one-pot procedure. Results show that changes in surface arrangements are enough to distinguish different volatile compounds and to recognize complex samples such as the headspace of cell cultures and vegetable oils [4].

A more recent exploitation of porphyrins grafted onto metal oxide nanostructures has been obtained for the development of an enantioselective QMB sensor [5]. The choice of QMB not only enables simple and fast sensors preparation but, more importantly, the non-selectivity of the mass transduction allows to explore the totality of the interactions occurring between the volatile compounds and the sensor, offering the opportunity to put in evidence the importance of chiral selective interactions respect to all the others.

Enantioselectivity is the capability of a sensor to show different sensitivities respect to chiral pairs of the same molecule. One of the main difficulties in chiral gas sensors is to transfer the chirality from single receptors to the solid-state material forming the sensing layer.

The molecular grafting on nanostructured material is a viable route to circumvent this problem. This property has been exploited by preparing a nanostructured film where chiral molecules are arranged on the surface of $\mathrm{ZnO}$ particles. The system retains chirality features and offers an optimal 
accessibility to the volatile compounds. We considered the inherently chiral porphyrin derivative where a (L)-proline unit infers the chiral features to a porphyrin macrocycle. Hydrothermally grown $\mathrm{ZnO}$ nanoparticles were functionalized in toluene solution of $\mathrm{ZnPL}$.

Figure 1 compares the response of sensors coated with chiral porphyrin and chiral porprhyrin grafted onto nanoparticles. Sensors were exposed to the enantiomers of S- and R-limonene, both at concentration of $375 \mathrm{ppm}$ in air. Figure 2 shows the sensor response as a function of concentration. The enantioselectivity is preserved in the whole investigated range of observation.

The sensing properties of layers of porphyrins onto $\mathrm{ZnO}$ nanostructures offer a further degree of freedom for the design of sensor arrays extending the properties and the capabilities of porphyrin based sensor array in particular for medical diagnosis, the quality and control of foods and the detection of compounds signaling harmful or dangerous substances.
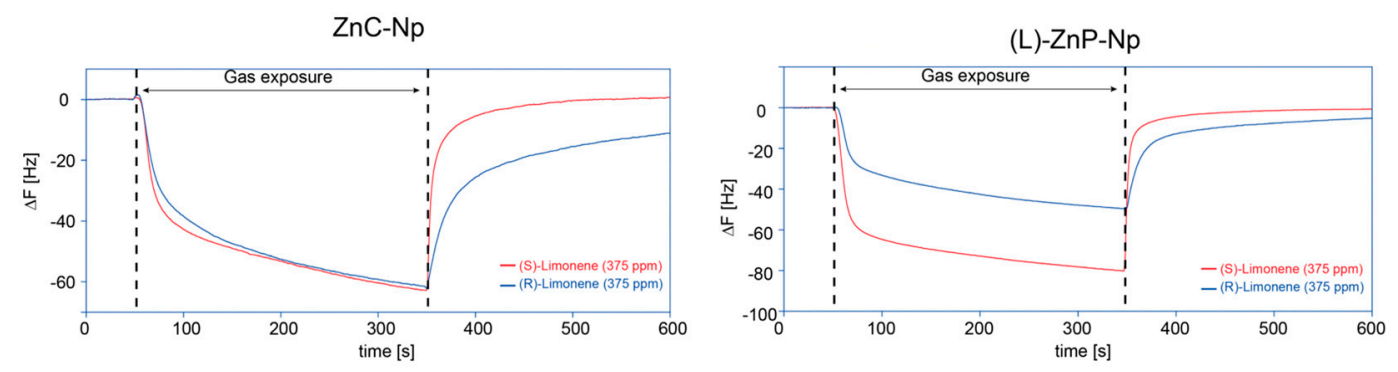

Figure 1. Time evolution of the sensor signal (change of QMB frequency) of chiral porphyrin on gold (left) and $\mathrm{ZnO}$ nanoparticles (right) exposed to the two enantiomers of limonene.
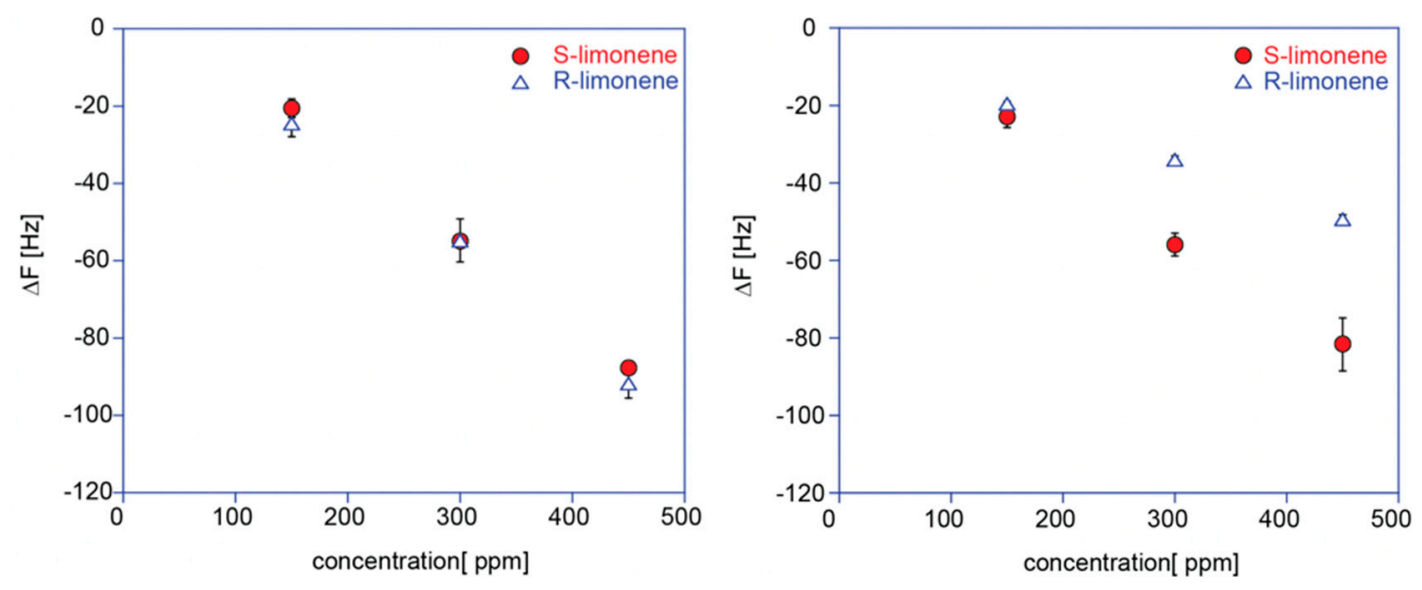

Figure 2. Response curve to limonene enantiomers of chiral porphyrin on gold (left) and $\mathrm{ZnO}$ nanoparticles (right).

\section{References}

1. Sivalingam, Y.; Martinelli, E.; Catini, A.; Magna, G.; Pomarico, G.; Basoli, F.; Paolesse, R.; Di Natale, C. GasSensitive Photoconductivity of Porphyrin-Functionalized ZnO Nanorods. J. Phys. Chem. C 2012, 116, 9151-9157.

2. Sivalingam, Y.; Magna, G.; Pomarico, G.; Catini, A.; Martinelli, E.; Paolesse, R.; Di Natale, C. The influence of gas adsorption on photovoltage in porphyrin coated ZnO nanorods. J. Mater. Chem. 2012, 22, 2003220037.

3. Magna, G.; Sivalingam, Y.; Martinelli, E.; Pomarico, G.; Basoli, F.; Paolesse, R.; Di Natale, C. The influence of film morphology and illumination conditions on the sensitivity of porphyrins-coated ZnO nanorods. Anal. Chim. Acta 2014, 810, 86-93.

4. Magna, G.; Zor, S.D.; Catini, A.; Capuano, R.; Basoli, F.; Martinelli, E.; Paolesse, R.; Di Natale, C. Surface arrangement dependent selectivity of porphyrins gas sensors. Sens. Actuators B 2017, 251, 524-532. 
5. Stefanelli, M.; magna, G.; Zurlo, F.; Caso, F.; di Bartolomeo, E.; Antonaroli, S.; Venanzi, M.; Paolesse, R.; Di Natale, C.; Monti, D. Chiral Selectivity of Porphyrin-ZnO Nanoparticle Conjugates. ACS Appl. Mater. Interfaces 2019, 11, 12077-122087, doi:10.1021/acsami.8b22749.

(c) (P)

(C) 2019 by the authors. Licensee MDPI, Basel, Switzerland. This article is an open access article distributed under the terms and conditions of the Creative Commons Attribution (CC BY) license (http://creativecommons.org/licenses/by/4.0/). 